\title{
Supporting Information of Bright plasmons with cubic nanometer mode volumes through mode hybridization
}

\author{
Tong $\mathrm{Wu}^{1}$, Wei Yan², Philippe Lalanne ${ }^{1, *}$ \\ ${ }^{1}$ LP2N, Institut d'Optique Graduate School, CNRS, Univ. Bordeaux, 33400 Talence, France \\ ${ }^{2}$ Institute of Advanced Technology, Westlake Institute for Advanced Study, 310024 Hangzhou, China \\ *philippe.lalanne@institutoptique.fr
}

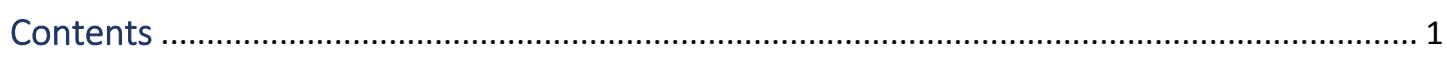

S1. The origin of the resonances of small protuberances and dips........................................ 2

S2. The mode volume of tiny spherical nanoresonators scales as $r^{3}$.................................. 4

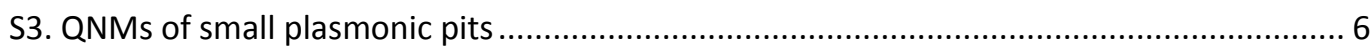

S4. Impact of quenching in the analytical gap-plasmon model................................................. 7

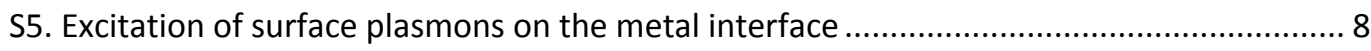

S6. Analysis of the impact of detuning on the performance of the hybrid cavity .................... 9

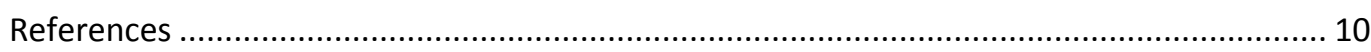




\section{S1. The origin of the resonances of small protuberances and dips}

In the main text, we show that a metallic protuberance on a metallic substrate is able to support resonances. This seems to be counter-intuitive at the first sight, because, unlike tiny plasmonic nanoparticles with two facets, e.g. nanorods, the protuberance is directly connected with a metallic substrate, therefore rising the question of whether the structure is able to support self-sustained free electron oscillations inside its volume. In this section, we show that the origin of resonances of atomicscale protuberance with a 'single' geometrical facet is the same as that of commonly seen tiny plasmonic nanoparticles with two facets; ${ }^{51}$ both are caused by Fabry-Perot resonances of plasmon waves.

Fabry-Perot resonance of the small metallic protuberance discussed in Figure 1 (main text) holds when the accumulated phase during a round trip is a multiple integer of $2 \pi, 2 n_{\mathrm{eff}} \operatorname{Re}\left(\tilde{k}_{0}\right) t+\phi_{r 1}+$ $\phi_{r 2}=2 \pi m$, where $m=1,2, \ldots, n_{\text {eff }}$ is the effective index of insulator-metal-insulator (IMI) plasmon, $\phi_{r 1,2}$ are the phases of the reflection $r_{1,2}$ at the terminations, and $\tilde{k}_{0}=\widetilde{\omega} / c$ is the complex wavenumber of the resonance mode (see the inset in Figure S1a for the shape of two kinds of terminations).

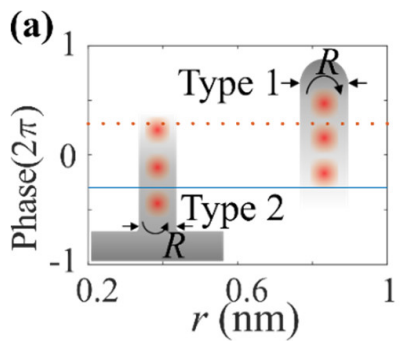

(b)

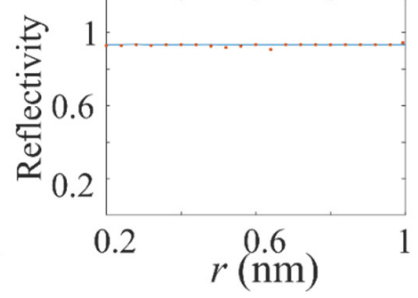

(c)

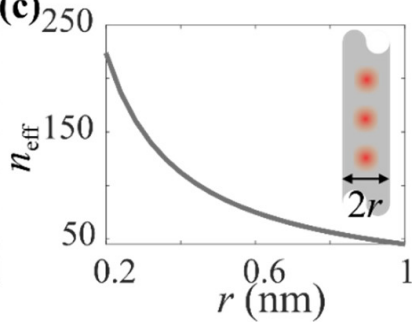

Figure S1. Fabry-Perot resonances of tiny protuberances. Phases (a) and amplitudes (b) of the reflection coefficients of the $\mathrm{Ag}$ nanowire with the termination of Type 1 (orange dotted line) and Type2 (blue solid line). (c) Effective index $n_{\text {eff }}$ of the Ag nanowire as a function of its radius $r$. The wavelength is $413 \mathrm{~nm}$; the permittivities of silver and air are taken as $\varepsilon_{A g}=-4.94+0.19 i$ and $\varepsilon_{b}=1$, respectively.

To understand how the important parameters in the Fabry-Perot model impact the resonances, we have computed the $\phi_{r 1}, \phi_{r 2}$, and $n_{\mathrm{eff}}$ as a function of the nanowire radius $r$. We first used the COMSOL RF module to numerically solve the reflection problem at the wire termination. The Ag nanowire is assumed to be semi-infinite and it is surrounded by an air background. Figures S1a-b show the phases $\left(\phi_{r 1,2}\right)$ and reflectivities caused by type 1 and type 2 terminations as a function of $r$ at a wavelength of $413 \mathrm{~nm}$. From Figure S1b, we find that the reflectivities at the two terminations are nearly identical and very close to 1 . This suggests that the mode lifetime of the small metallic protuberance is not limited by radiation into the metallic substrate but by absorption.

Another important parameter is the nanowire effective index $n_{\text {eff }}$. The latter, shown in Figure S1c, is inversely proportional to $r$. The large increase of $n_{\text {eff }}$ as $r$ decreases is a key ingredient for building ultra-small resonators since the resonator length $t$, which scales as $\lambda_{0} / 2 n_{\text {eff }}$ according to the FabryPerot condition, becomes proportional to $r$.

In the main text, we already verified that the Fabry-Perot-model well predicts the resonance frequencies of the protuberance computed with the QNMEig solver. ${ }^{S 2}$ Here, for completeness, we 
further verify that the model is also valid for nanorods consisting of a cylindrical part with height $t$ and two hemispherical terminations, see the inset of Figure S2b. The Fabry-Perot-model predictions, shown in Figure S2b with solid curves quantitatively agree with full-wave computational results (dots) obtained with QNMEig. Unlike the protuberance case, the zero-order mode $(m=0)$ does not exist because the reflection of the hemispherical termination contributes a positive phase (Figure S1a). The lowest order mode $m=1$ (Figure S2a) bears a uniform distribution, behaving as a dipole polarized along the $z$-axis, which is in analogy to the $m=0$ mode of the small protuberance.

(a)

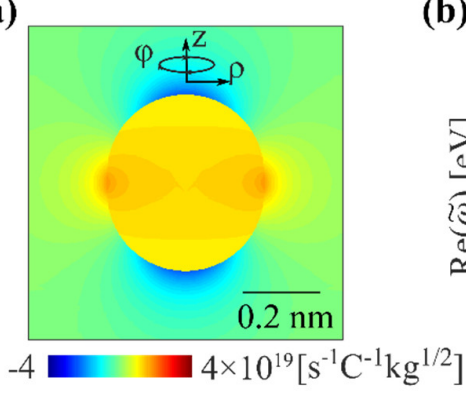

(b)

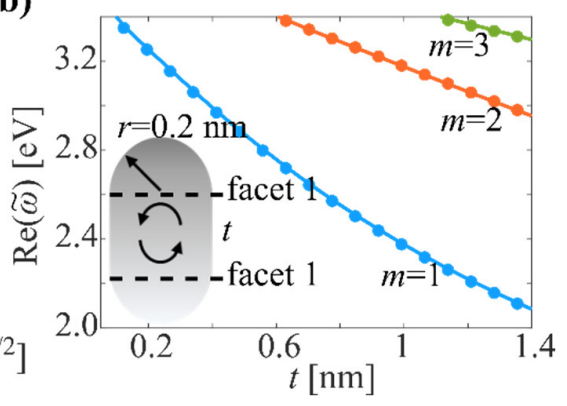

Figure S2. Resonance QNMs of small metallic nanorods. (a) Electric-field z-component, $\operatorname{Re}\left(\tilde{E}_{z}\right)$, of the fundamental electrical-dipole QNM of an atomic-scale Ag nanorod in air $\left(\varepsilon_{b}=1\right)$. The rod radius and height are $r=0.2 \mathrm{~nm}$ and $t=0.1 \mathrm{~nm}$. The mode volume $\tilde{V}=1 /\left(2 \varepsilon_{0} \varepsilon_{b} \tilde{E}_{z}^{2}\right)$ reaches a value as small as $0.15 \mathrm{~nm}^{3}$ close to the apex of the cylinder. (b) A classical Fabry-Perot model (solid curves) quantitively predicts the three first QNM branches computed with full-wave simulations (dots). The silver permittivity $\varepsilon_{A g}$ is given in the caption of Figure 1 of the main text. 


\section{S2. The mode volume of tiny spherical nanoresonators scales as $r^{3}$}

In this Section, we theoretically show that the mode volumes of the dipolar QNMs of metal nanospheres scale with their geometric volumes in the limit of small radii. Albeit intuitive, this result needs to be carefully demonstrated owing to the exponential growth of QNM fields outside the resonator, even in the static limit.

We consider a metallic sphere of radius $r(r \ll \lambda)$. According to the electrostatic approximation, the sphere polarizability is

$$
\alpha(\omega)=4 \pi r^{3} \varepsilon_{0}\left[\frac{\varepsilon(\omega)-\varepsilon_{b}}{\varepsilon(\omega)+2 \varepsilon_{b}}\right],
$$

where $\varepsilon(\omega)$ and $\varepsilon_{b}$ are the sphere and background permittivities. We adopt a Drude local-response dielectric function for the metal

$$
\varepsilon(\omega)=\varepsilon_{\infty}-\frac{\omega_{p}^{2}}{\omega^{2}+i \omega \gamma}
$$

where $\omega_{p}$ is the plasma frequency, $\gamma$ is the damping coefficient, and $\varepsilon_{\infty}$ is the permittivity at $\omega=\infty$. The dipolar resonance frequency $\widetilde{\omega}$ for the small sphere can be obtained by solving $\alpha^{-1}(\omega)=0$, and is given by

$$
\widetilde{\omega}=\frac{\sqrt{4 \omega_{p}^{2}-\gamma^{2}\left(\varepsilon_{\infty}+2 \varepsilon_{b}\right)}}{2 \sqrt{\varepsilon_{\infty}+2 \varepsilon_{b}}}-i \frac{\gamma}{2}
$$

With a first-order Taylor expansion of $\alpha^{-1}(\omega)$ at $\widetilde{\omega}$, the polarizability near the resonance frequency can be approximated as

$$
\alpha(\omega \sim \widetilde{\omega}) \approx \frac{1}{\omega-\widetilde{\omega}} \frac{-9 V \varepsilon_{b} \varepsilon_{0} \omega_{p}^{2}}{\left(\varepsilon_{\infty}+2 \varepsilon_{b}\right)^{\frac{3}{2}} \sqrt{4 \omega_{p}^{2}-\gamma^{2}\left(\varepsilon_{\infty}+2 \varepsilon_{b}\right)}}=\frac{\alpha_{0} V \varepsilon_{0} \omega_{p}}{\omega-\widetilde{\omega}},
$$

where $V=4 \pi r^{3} / 3$ is the volume of the sphere, $\alpha_{0}=-9 \varepsilon_{b} \omega_{p} /\left[2(\operatorname{Re} \widetilde{\omega})\left(\varepsilon_{\infty}+2 \varepsilon_{b}\right)^{2}\right]$ is a dimensionless coefficient that depends only on the material and is of the order of $O(1)$.

To normalize the mode, the critical step, we follow the approach in $\mathrm{Bai}^{\mathrm{S3}}$ and consider a dipole source $\mathbf{p}$ near the sphere. If the source is emitting at a frequency $\omega \approx \widetilde{\omega}$, the system response is dominated by the excitation of the dipolar QNM. The field scattered by the sphere (this is also the total field for $\omega \sim \widetilde{\omega})$ is therefore ${ }^{\varsigma 4}$

$$
\mathbf{E}_{s}(\mathbf{r}, \omega)=\frac{\omega}{\widetilde{\omega}-\omega}\left[\tilde{\mathbf{E}}\left(\mathbf{r}_{0}\right) \cdot \mathbf{p}\right] \tilde{\mathbf{E}}(\mathbf{r}),
$$

where $\tilde{\mathbf{E}}$ is the normalized dipolar QNM and $\mathbf{r}_{0}$ is the position of the dipole source. Multiplying eq S5 by $\mathbf{p}$ and setting $\mathbf{r}=\mathbf{r}_{0}$, one can readily obtain $\mathbf{p} \cdot \tilde{\mathbf{E}}\left(\mathbf{r}_{\mathbf{0}}\right)=\sqrt{(\widetilde{\omega}-\omega) \mathbf{p} \cdot \mathbf{E}_{S}\left(\mathbf{r}_{0}, \omega\right) / \widetilde{\omega}}$. Introducing this value into eq $\mathrm{S} 5$, we obtain an expression for the normalized mode $\mathrm{S}^{\mathrm{S3}}$

$$
\tilde{\mathbf{E}}(\mathbf{r})=\lim _{\omega \rightarrow \widetilde{\omega}} \sqrt{\frac{\widetilde{\omega}-\omega}{\omega \mathbf{p} \cdot \mathbf{E}_{S}\left(\mathbf{r}_{0}, \omega\right)}} \mathbf{E}_{S}(\mathbf{r}, \omega) \text {. }
$$

On the other hand, $\mathbf{E}_{S}(\mathbf{r})$ can also be computed by using the polarizability of the sphere $\alpha(\omega)$,

$$
\mathbf{E}_{S}(\mathbf{r}, \omega)=\alpha(\omega) \overline{\overline{\mathrm{G}}}_{0}\left(\mathbf{r}, \mathbf{r}_{s}\right) \cdot\left[\overline{\overline{\mathrm{G}}}_{0}\left(\mathbf{r}_{s}, \mathbf{r}_{0}\right) \cdot \mathbf{p}\right]
$$


where $\overline{\overline{\mathrm{G}}}_{0}\left(\mathbf{r}, \mathbf{r}^{\prime}\right)$ is the dyadic's Green function in a uniform medium with the permittivity $\varepsilon_{b}$ and $\mathbf{r}_{s}$ is the position of the small metallic sphere.

Associating eqs $\mathrm{S} 4, \mathrm{~S} 6$ and $\mathrm{S} 7$ and using the reciprocity theorem of the green function $\overline{\overline{\mathrm{G}}}_{0}\left(\mathbf{r}_{s}, \mathbf{r}_{0}\right)=$ $\overline{\overline{\mathrm{G}}}_{0}^{\mathrm{T}}\left(\mathbf{r}_{0}, \mathbf{r}_{s}\right)$, the normalized electric field for the $k$ polarized dipolar QNM ( $k$ stands for $x, y$ or $z$ ) of the small metallic sphere is given by

$$
\tilde{\mathbf{E}}(\mathbf{r})=\sqrt{-\frac{\omega_{p}}{\widetilde{\omega}}} \sqrt{\alpha_{0} V \varepsilon_{0}} \overline{\overline{\mathrm{G}}}_{0}\left(\mathbf{r}, \mathbf{r}_{\mathrm{s}}\right) \cdot \mathbf{e}_{k}
$$

Equation $\mathrm{S} 8$ is the expression of the normalized dipolar field of a tiny sphere in the static limit. The field diverges away from the sphere since the frequency is complex. Taking the z-polarized QNM component for example, in the near-field regime, $\overline{\bar{G}}_{0}\left(\mathbf{r}, \mathbf{r}_{\mathrm{s}}\right) \cdot \mathbf{e}_{z} \approx \frac{\mu_{0}}{4 \pi}\left(-\mathbf{e}_{z} \frac{1}{\varepsilon_{b} \varepsilon_{0} \mu_{0} R^{3}}+\right.$ $\left.\frac{\mathbf{r}-\mathbf{r}_{S}}{R} \frac{z-z_{S}}{R} \frac{3}{\varepsilon_{b} \varepsilon_{0} \mu_{0} R^{3}}\right)$. The mode volume is given by

$$
\tilde{V}_{z}(\mathbf{r})=\left[2 \varepsilon_{0} \varepsilon_{b} \widetilde{E}_{z}^{2}\right]^{-1}=-\frac{\varepsilon_{b} \widetilde{\omega}\left(V^{\prime}\right)^{2}}{2 \alpha_{0} \omega_{p} V}\left(-\frac{1}{3}+\frac{\left(z-z_{S}\right)^{2}}{R^{2}}\right)^{-2},
$$

where $V^{\prime}=\frac{4}{3} \pi R^{3}$ and $R=\left|\mathbf{r}-\mathbf{r}_{s}\right|$.

To numerically verify the formula eq $\mathrm{S} 9$, we compute the mode volume distribution of a small sphere of $r=1 \mathrm{~nm}$. Figure S3 compares the maps of $\operatorname{Re}\left(1 / \tilde{V}_{Z}\right)$ computed with eq S9 and with QNMEig ${ }^{\mathrm{S2}}$. A perfect agreement is achieved.
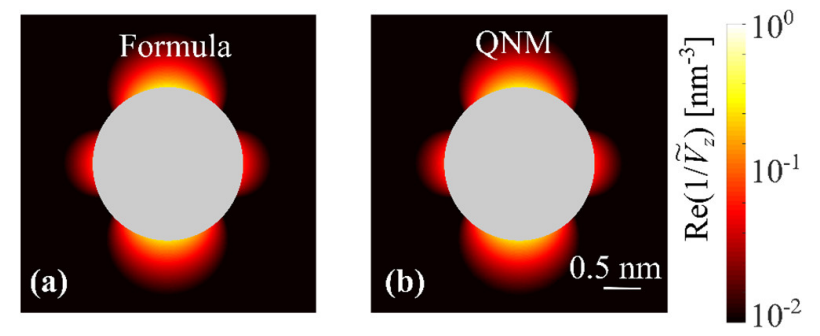

Figure S3. Maps of $\operatorname{Re}\left(1 / \tilde{V}_{z}\right)=\operatorname{Re}\left(2 \varepsilon_{0} \varepsilon_{b} E_{z}^{2}\right)$ for the QNM of a metallic nanosphere embedded in air $\left(\varepsilon_{b}=1\right)$ for a radius $r=1 \mathrm{~nm}$. The permittivity of the sphere $\varepsilon(\omega)$ is given by a Drude model with $\varepsilon_{\infty}=1, \omega_{p}=1.37 \times 10^{16}$ and $\gamma=0.0023 \omega_{p}$. The QNM shown here corresponds to the $z$-polarized dipolar mode. (a) Map computed directly from eq S9; (b) Map computed with QNMEig.

Considering that the factor $-\frac{\varepsilon_{b} \widetilde{\omega} V^{\prime}}{2 \alpha_{0} \omega_{p} V}$ and $\left(-\frac{1}{3}+\frac{\left(z-z_{s}\right)^{2}}{R^{2}}\right)^{-2}$ in eq $S 9$ are both dimensionless and of the order of $O(1)$, we conclude that the mode volume scales with the geometrical sphere volume. Thus, near the surface of small metallic spheres, $\tilde{V}$ is of the order of $r^{3}$. For the simplest case, where $\varepsilon_{\infty}=1, \varepsilon_{b}=1$ and $\widetilde{\omega}_{p} \gg \gamma$, the minimum volume $\tilde{V}_{\text {min }}=\pi r^{3}$ occurs at the sphere apex, $\mathbf{r}-\mathbf{r}_{s}=$ $(0,0, r)$. 


\section{S3. QNMs of small plasmonic pits}

In the main text, we have verified that small plasmonic protuberances on metal surfaces are able to support resonances with ultra-small mode volumes. In this section, we discuss the resonances of another kind of roughness, small plasmonic pits. The pit shown in the inset of Figure S4b can be viewed as the complementary structure of the protuberance.

Figure S4a shows the normalized electric-field z-component, $\operatorname{Re}\left(\tilde{E}_{z}\right)$, of the QNM dominating the scattering of a pit with a depth $t=0.2 \mathrm{~nm}$ and aperture $r=0.2 \mathrm{~nm}$. The field computed with QNMEig is strongly localized at the edges of the pit with a mode volume smaller than $1 \mathrm{~nm}^{3}$. Similar to other plasmonic resonators, the ultra-small mode volume of the present structure is also due to its small size. As shown in Figure $\mathrm{S} 4 \mathrm{~b}$, the real part of the complex mode volume $\operatorname{Re}(\tilde{V})$ increases linearly with the geometrical volume $V$ of the pit.
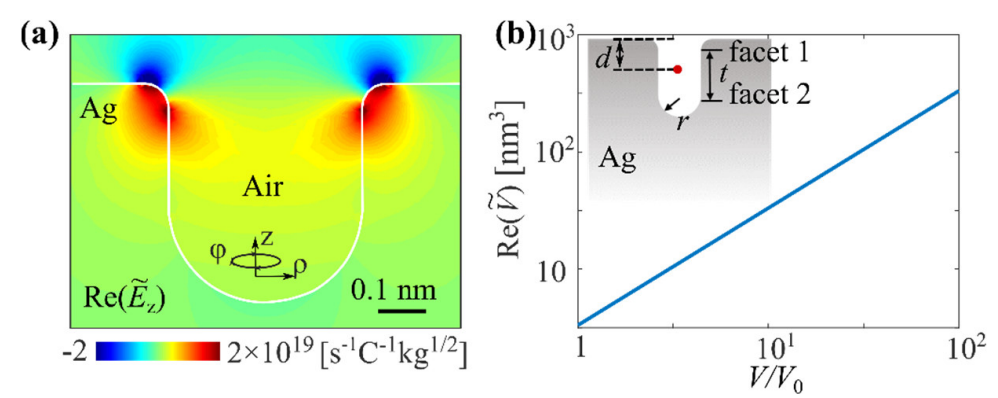

Figure S4. Mode volume of small pits. (a) Electric-field z-component, $\operatorname{Re}\left(\tilde{E}_{z}\right)$, of the fundamental QNM of an atomic-scale pit on an Ag substrate. The mode has a resonance frequency $\operatorname{Re}(\widetilde{\omega})=$ $3.63 \mathrm{eV}$ and a quality factor $Q=26$. (b) $\operatorname{Re}(\tilde{V})$ at a point (red dot) located at a distance $d$ below the pit mouth. The geometric parameters for the system are given by $r=0.2 f \mathrm{~nm}, d=0.15 f \mathrm{~nm}$ and $t=0.2 f \mathrm{~nm}$ with $f=\left(V / V_{0}\right)^{1 / 3}, V_{0}$ being the reference pit volume for $r=0.2 \mathrm{~nm}, d=$ $0.15 \mathrm{~nm}$ and $t=0.2 \mathrm{~nm}$. The pit edge is chamfered with a $0.05 f \mathrm{~nm}$ radius curvature. Inset: Illustration diagram of the pit on an infinite Ag substrate. The frequency and quality factor of the pit are left unchanged as $r$ and $t$ are proportionally scaled. The silver permittivity $\varepsilon_{A g}$ is given in the caption of Figure 1 of the main text. 


\section{S4. Impact of quenching in the analytical gap-plasmon model}

To derive the analytical formula eq 1 in the main text, we ignore the quenching $\Gamma_{\text {quench }}$ of the electric dipole of the protuberance caused by the MIM top interface, leading to a difference which can be as large as $30 \%$ between the result of the analytical model and that of numerical calculation. Here, we reincorporate this term into the analytical formula to see whether it affects the conclusion in the main text. Defining the ratio of $\Gamma_{\text {quench }}$ to $\Gamma_{N R}$ as $\rho$, the $\beta$-factor is thus revised as

$$
\beta \approx \frac{\Gamma_{g s p}}{\Gamma_{N R}+\Gamma_{\text {quench }}+\Gamma_{g s p}}=\left[1+\frac{(1+\rho) g^{3} \operatorname{Im}\left(\varepsilon_{m}\right)\left|\varepsilon_{m}\right|^{2}}{2\left|\varepsilon_{m}-\varepsilon_{b}\right|^{2} \varepsilon_{b} V_{a}}\right]^{-1} .
$$

In general, $\rho$ depends on many factors, e.g. permittivities, protuberance shape, gap size, and cannot be derived analytically. However, since the induced electric dipole of the protuberance is much closer to the upper interface compared to the lower interface, it is reasonable to assume that $\rho$ weakly depends on the gap size $g$. We further find empirically that, when we fix the aspect ratio of the protuberance, $\rho$ remains unchanged as we vary the protuberance geometrical volume $V_{a}$. For the protuberance discussed in Figure 3 (main text), with an aspect ratio of $a / b=1.91, \rho \approx 0.52$. This value is obtained by computing the total decay rate $\gamma^{(0)}$ and absorption rate of the protuberance $\Gamma_{N R}^{(0)}$ in the absence of the lower MIM interface, $\rho=\left(\gamma^{(0)}-\Gamma_{N R}^{(0)}\right) / \Gamma_{N R}^{(0)}$. The solid lines in Figure $\mathrm{S} 5$ show $\beta$ as a function of $g$ (computed with eq S10) for protuberances of different sizes. Compared to the results by eq 1 in the main text, it is found that the results by eq $\mathrm{S} 10$ have a better agreement with those from numerical computation (the dotted lines). Thus, by setting $\rho$ as a constant value, we can perfectly account for the influence of $\Gamma_{\text {quench }}$ on the $\beta$, and the conclusion in the main text that $\beta$ is dominated by the ratio $g^{3} / V_{a}$ still holds up.

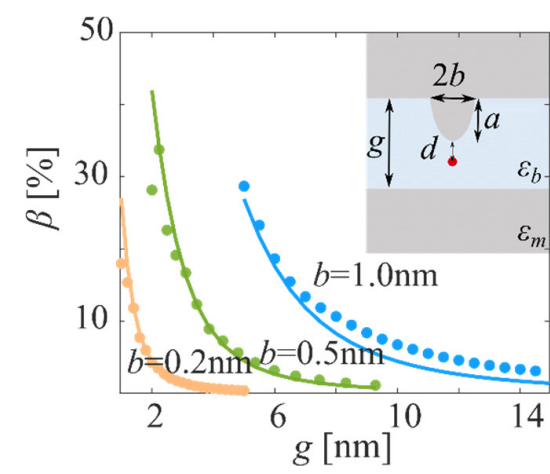

Figure S5. Fully vectorial calculations and analytical predictions from eq S10 are shown with markers and solid curves, respectively. In the vectorial calculation, a vertically polarized dipole emitter is placed below the protuberance with a distance of $d=b$. The analytical formula is independent with the position of the emitter. Inset: The protuberance is modeled as a demi-spheroid with $a=1.91 b$. Such an aspect ratio leads to the ratio $\rho=\Gamma_{\text {quench }} / \Gamma_{N R}=0.52$. The permittivities of the gap and silver are taken as $\varepsilon_{b}=2.1$ and $\varepsilon_{m}=$ $-9.65+0.24 i$, respectively. The emission frequency is taken as $2.17 \mathrm{eV}$. 


\section{S5. Excitation of surface plasmons on the metal interface}

In Figure 4 of the main text, the radiative efficiency $\eta_{p h}$ is defined as the fraction of the total power emitted by the source which is funneled into free-space photons, $\eta_{p h}=\gamma_{p h} / \gamma$ with $\gamma$ the total decay rate and $\gamma_{p h}$ the decay rate into photons.

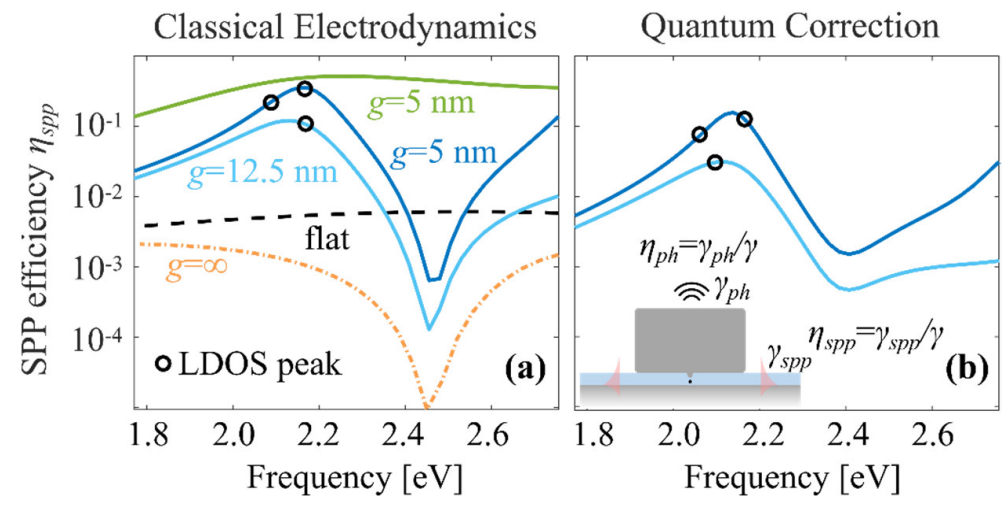

Figure S6. Analogue of Figure 4 for SPPs launched on the metal surface. (a) SPP generation efficiency $\eta_{s p p}$ for $g=5 \mathrm{~nm}$ and $12.5 \mathrm{~nm}$ (blue curves). The different geometries are described in the caption of Figure 4. The black circles indicate the peaks of the LDOS. (b) Same as in (a) with non-classical quantum surface corrections introduced with the Feibelman's d-parameters.

However, surface plasmon polaritons (SPPs) are also launched on the metal surface, and with the help of a cylindrical grating, they might be converted into photons. Computational results of the SPP generation efficiency, $\eta_{s p p}=\gamma_{s p p} / \gamma$, are shown in Figures $\mathrm{S} 6 \mathrm{a}$ and $\mathrm{b}$. They are obtained by using the freeware package RETOP, ${ }^{55}$ which allows to compute far-field radiation diagrams into guided waves. The frequency-dependence trends of $\eta_{s p p}$ follow those of $\eta_{p h}$ presented in Figure 4. $\eta_{s p p}$ for a protuberance on a single interface $(g=\infty)$ is extremely small, even weaker than that obtained for the same emitter placed at a $1 \mathrm{~nm}$ distance from a flat interface. Hybrid cavities exhibit much larger values. For the cavity with $g=12.5 \mathrm{~nm}, \eta_{s p p}$ at the resonance frequency (shown with the black circle) is $\approx$ $10 \%$. By shrinking the width to $g=5 \mathrm{~nm}, \eta_{s p p}$ can reach $\approx 30 \%$, a value comparable to the efficiencies of the NPoM without the protuberance (green solid line in Figure S6a). Quantum corrections (Figure S6b) only moderately impact our conclusions. The slight efficiency decrease can be attributed to the fact that surface-assisted Landau damping decreases the modal field intensity inside the gap, therein weakening the nanocavity capability to funnel the energy from the protuberance to the gap plasmons. 


\section{S6. Analysis of the impact of detuning on the performance of the hybrid cavity}

In Figure 4 of the main text, we assume that the frequencies of the NPoM and protuberance modes are perfectly matched. However, since in practice the precise size of the protuberance is difficult to control, ${ }^{56,57}$ the modes are likely not be matched. In this Section, we address the impact of frequency detuning and show that high radiative efficiencies and tight optical confinements are still achievable for significant mismatches.

We again consider the two hybrid cavities of Figure 4 with $g=5 \mathrm{~nm}$ and $g=12.5 \mathrm{~nm}$, and vary the protuberance aspect ratio $a / b$ for a fixed value $b=1 \mathrm{~nm}$ to tune the frequency of the protuberance mode. Figures $\mathrm{S7} a$ and $b$ show the free-space radiative efficiencies and LDOS for the $g=$ $5 \mathrm{~nm}$ case. When the frequencies are matched $(a / b=1.9)$, the LDOS spectrum presents two peaks which are caused by the excitation of the two hybrid modes. As we tune the protuberance frequency by changing its height by $\pm 16 \%$, the coupling is lowered and the efficiency $\eta_{p h}$ at the resonance frequencies (labeled with circles) is also slightly lowered but remains significant with a value of 3-4\%. The brightness of the hybrid-cavity with large gaps is resilient to detuning. For the $g=12.5 \mathrm{~nm}$ (Figures S7c and d), the NPoM and protuberance modes are less coupled and only a single peak due to the excitation of the protuberance-like mode is observed in the LDOS spectra. The efficiency $\eta_{p h}$ is weakly lowered from $2.5 \%$ to $1 \%$ and $2 \%$ for $a / b=1.6$ and 2.2 , respectively. Such an efficiency remains three orders of magnitude larger compared to that of the dark mode of an isolated protuberance (see the dashed line in Figure 4c).
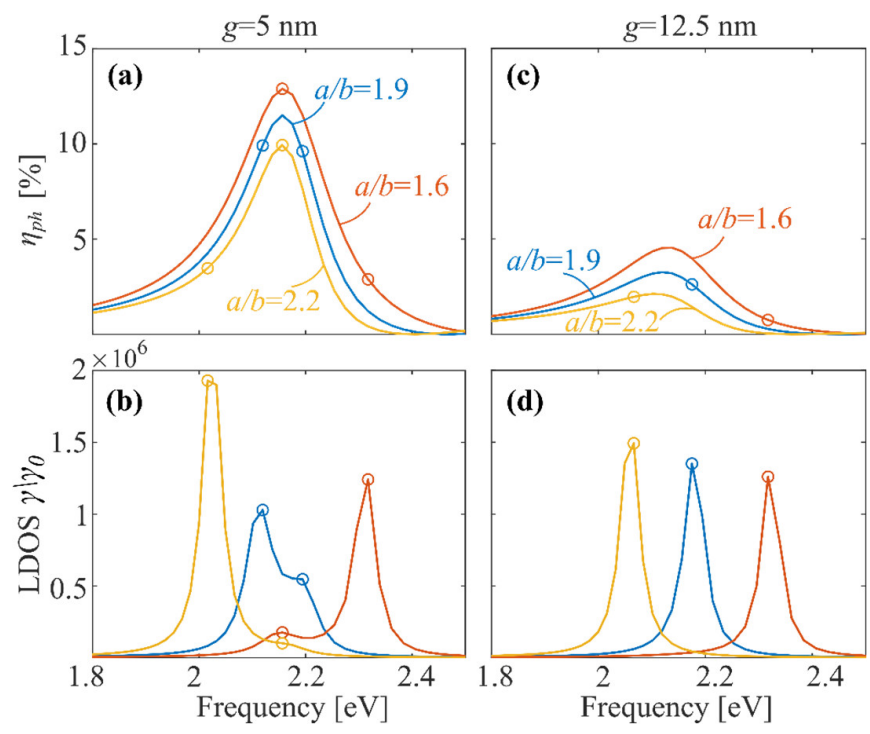

Figure S7. Tolerance of the hybrid cavities against detuning. (a-b) Free-space radiative efficiencies $\eta_{p h}$ and normalized decay rates $\gamma / \gamma_{0}$ for $g=5 \mathrm{~nm}$. The dipole emitter is placed $1 \mathrm{~nm}$ away from the protuberance apex. The protuberance is modeled as a half-spheroid with long and short axes $a$ and $b$. The protuberance aspect ratio $a / b$ is varied for a fixed value $b=1 \mathrm{~nm}$ to control the mode detuning. Three different aspect ratios are considered, $a / b=1.6,1.9,2.2$. The circles denote the peaks of the LDOS spectra. (c-d) Same as (a-b) but for $g=12.5 \mathrm{~nm}$. 


\section{References}

S1. Hasan, S. B.; Filter, R.; Ahmed, A.; Vogelgesang, R.; Gordon, R.; Rockstuhl, C.; Lederer, F. Relating localized nanoparticle resonances to an associated antenna problem. Phys. Rev. B: Condens. Matter Mater. Phys. 2011, 84, 195405.

S2. QNM solvers and toolboxes are freely available online at https://www.lp2n.institutoptique.fr Llight-complex-nanostructures.

S3. Bai, Q.; Perrin, M.; Sauvan, C.; Hugonin, J.-P.; Lalanne, P. Efficient and intuitive method for the analysis of light scattering by a resonant nanostructure. Opt. Express 2013, 21, 27371-27382.

S4. Sauvan, C.; Hugonin, J. P.; Maksymov, I. S.; Lalanne, P. Theory of the spontaneous optical emission of nanosize photonic and plasmon resonators. Phys. Rev. Lett. 2013, 110, 237401.

S5. Yang, J.; Hugonin, J. P.; Lalanne, P. Near-to-far field transformations for radiative and guided waves. ACS photonics 2016, 3, 395-402. The RETOP freeware is available at the corresponding author group webpage..$^{.2}$

S6. Yang, B.; Chen, G.; Ghafoor, A.; Zhang, Y.; Zhang, Y.; Zhang, Y.; Luo, Y.; Yang, J.; Sandoghdar, V.; Aizpurua, J.; Dong, Z.-C.; Hou, J. G. Sub-nanometre resolution in single-molecule photoluminescence imaging. Nat. Photonics 2020, 14, 693-699.

S7. Carnegie, C.; Griffiths, J.; de Nijs, B.; Readman, C.; Chikkaraddy, R.; Deacon, W. M.; Zhang, Y.; Szabb, I.; Rosta, E.; Aizpurua, J.; Baumberg, J. J. Room-Temperature Optical Picocavities below 1 $\mathrm{nm}^{3}$ Accessing Single-Atom Geometries. J. Phys. Chem. Lett. 2018, 9, 7146- 7151. 